\title{
RENILLA LUCIFERASE AS A REPORTER TO ASSESS SARS-COV mRNA TRANSCRIPTION REGULATION AND EFFICACY OF ANTI-SARS-CoV AGENTS
}

\author{
Rhonda S. Roberts, Boyd L. Yount, Amy C. Sims, Susan Baker, and \\ Ralph S. Baric*
}

\section{INTRODUCTION}

SARS coronavirus (SARS CoV) is the etiologic agent responsible for the pandemic of SARS. ${ }^{1}$ We have developed a reverse genetics model to characterize the pathways of replication and pathogenesis of this virus. ${ }^{2}$ Renilla Luciferase was used as a reporter gene and inserted into the backbone of the infectious clone of SARS-CoV to replace ORF $7 \mathrm{a} / \mathrm{b}$ (SARS wt-Luc), which is believed to have apoptotic effects on host cells. Subsequently, to measure the impact of the transcription regulatory sequence (TRS) on gene expression, either two or three mutations were introduced into the ORF 7a/b TRS hexanucleotide site of the luciferase cassette; SARS 2mut-Luc and SARS 3mut-Luc, respectively (Figure 1).

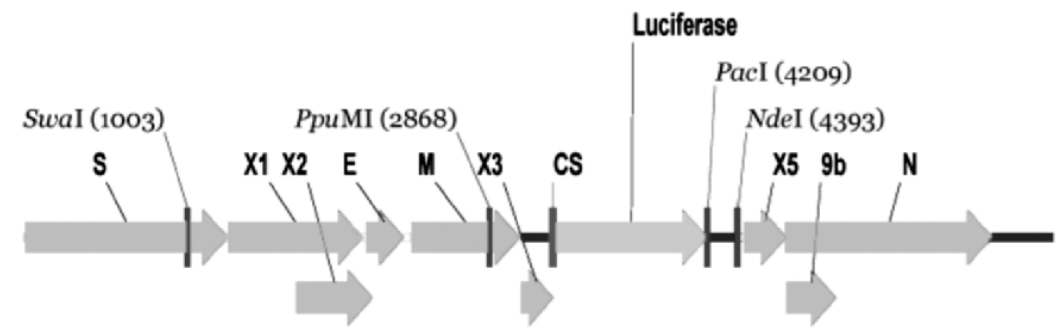

Figure 1. Structure of SARS wt-Luc and introduction of mutations into the TRS site to create the SARS 2mutLuc and SARS 3mut-Luc viruses.

*Rhonda S. Roberts, Boyd L. Yount, Amy C. Sims, Ralph S. Baric, University of North Carolina, Chapel Hill, North Carolina 27599. Susan Baker, Loyola University Medical Center, Maywood, Illinois 60153. 


\section{RESULTS AND DISCUSSION}

Recombinant viruses with all three Luciferase constructs were isolated and shown to stably maintain the Renilla Luciferase gene and to express subgenomic mRNA encoding luciferase. The growth kinetics of all three constructs was similar, suggesting that the TRS mutations did not effect the efficacy of virus replication. However, Western blot analysis detected Luciferase protein in the SARS wt-Luc construct but did not detect the expression of any Luciferase protein in cells infected with the 2 mut and 3 mut recombinant viruses. Likewise, Northern blot analysis exhibited a severely decreased amount of subgenomic Luciferase mRNA in cells infected with the two mutant constructs (data not shown). Combined, these results effectively demonstrate that the TRS mutations attenuate the virus' ability to efficiently produce or translate subgenomic mRNA. This analysis was verified utilizing the Dual-Glo Luciferase Assay System kit (Promega) to detect Luciferase enzyme activity. The Luciferase expression, measured in relative light units (RLU), decreased from almost $1 \log$ to approximately $1.5 \operatorname{logs}$ in cells infected with the 2 mut and 3 mut constructs, respectively (Figure 2).

Sequence analysis of the mutant viruses suggested that the reduced Luciferase expression was due to a mechanism used to bypass the mutations in the TRS region. There were various noncanonical leader body-junctions identified surrounding the TRS region in the subgenomic sequence in the two mutant constructs. Replicase slippage and the usage of noncanonical TRS-like junctions allowed for low level subgenomic transcription and expression of Luciferase.

The recombinant SARS wt-Luc virus was also used to measure the efficacy of putative anti-SARS agents. Four various interferon (IFN) treatments were tested in their ability to protect host cells against SARS infection: IFN- $\alpha 2$, IFN- $\beta$, IFN- $\gamma$, and a mixture of IFN- $\beta$ plus IFN- $\gamma$. CaCo2 cells were seeded at an approximate concentration of

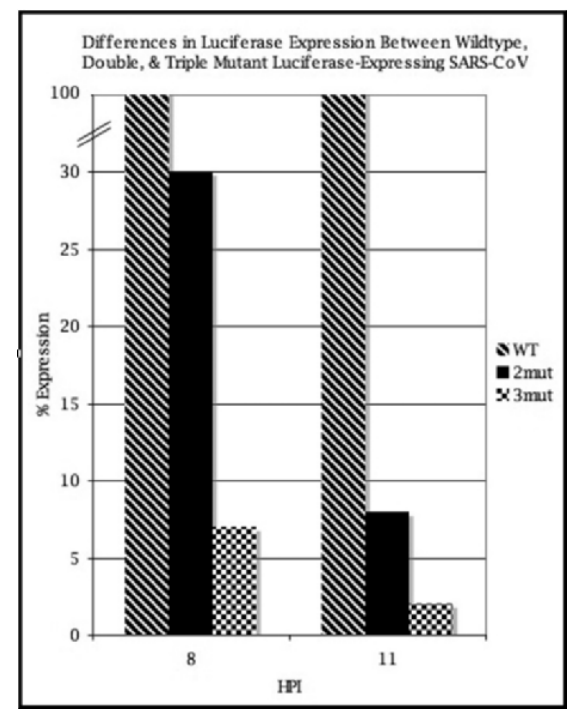

Figure 2. Decrease in Luciferase expression observed in cells infected with the SARS 2mut-Luc and SARS 2mut-Luc viruses compared with SARS wt-Luc. 
$2 * 10^{5}$ cells/ well on a 96 -well plate 24 hours prior to infection. At 8 hours prior to infection, 0-200 international units (U) of the appropriate IFN treatment was added to whole media. Cells were infected with SARS wt-Luc at a multiplicity of infection (MOI) of 0.5 and allowed to incubate at room temperature for 1 hour. Luciferase expression was measured at various time points for 24 hours postinfection (HPI). While all IFN treatment effectively inhibited SARS infection by at least 2 logs, the synergistic effect of IFN$\beta /$ IFN- $\gamma$ had the most extreme effect at $200 \mathrm{U}$,decreasing luciferase activity by more than $3 \operatorname{logs}$ or more than $98 \%$ (Figure 3 ).

In addition to IFN treatment, synthetic chemical compounds were developed to inhibit MPro binding; 0309, 0310, 0312, and 0313. Vero E6 cells were seeded approximately 24 hours prior to infection as described above. They were infected with SARS wt-Luc at a MOI of 0.5 and allowed to incubate for 1 hour at room temperature. Subsequently, $150 \mu \mathrm{l}$ of whole media with $100 \mu \mathrm{m}$ of the appropriate drug was added. Luciferase expression was assessed via the luciferase bioassay at various time points up to $40 \mathrm{HPI}$. Each drug decreased luciferase expression by at least $40 \%$ (Figure 4). AntiSARS agent 0310 was then used in subsequent effective dose studies. Vero E6 cells were initially treated in the same manner except, after the 1 hour infection period, $150 \mu \mathrm{l}$ media with 0310 diluted at various concentrations, ranging from $6.25 \mu \mathrm{M}$ to $200 \mu \mathrm{M}$, was added to each well. Again, the Luciferase expression was measured until 31 HPI. 0310 exhibited a small dose-response with $6.25 \mu \mathrm{M}$ decreasing Luciferase activity $1 \mathrm{log}$ and increasing concentrations thereafter showing enhanced activity, with an almost $2 \mathrm{log}$ reduction at a concentration of 0310 of $200 \mu \mathrm{M}$ (data not shown).

We demonstrate that SARS wt-Luc is a viable virus that allows for studies of the effect of subgenomic manipulation on virus efficacy, both in replication and subgenomic

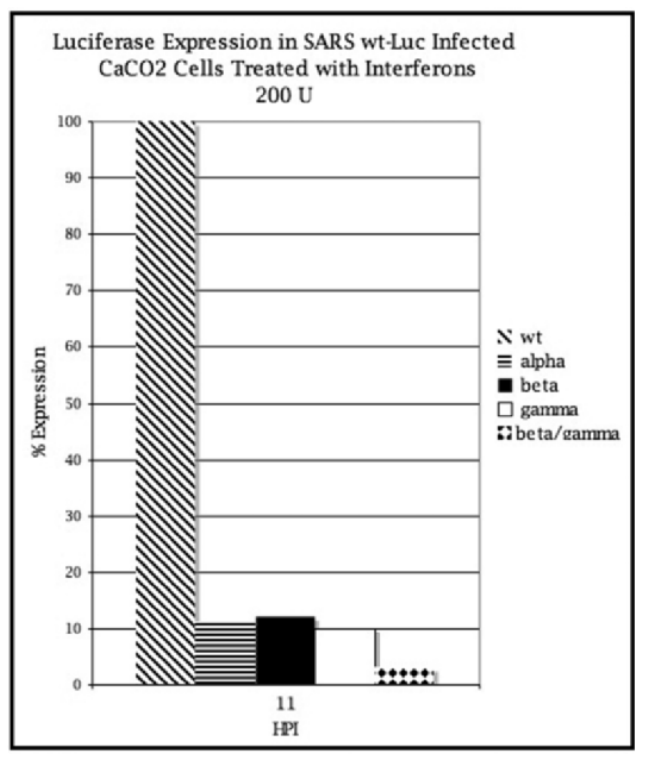

Figure 3. Luciferase expression in SARS wt-Luc infected $\mathrm{CaCo} 2$ cells after being pretreated with various interferons at $200 \mathrm{U}$. 


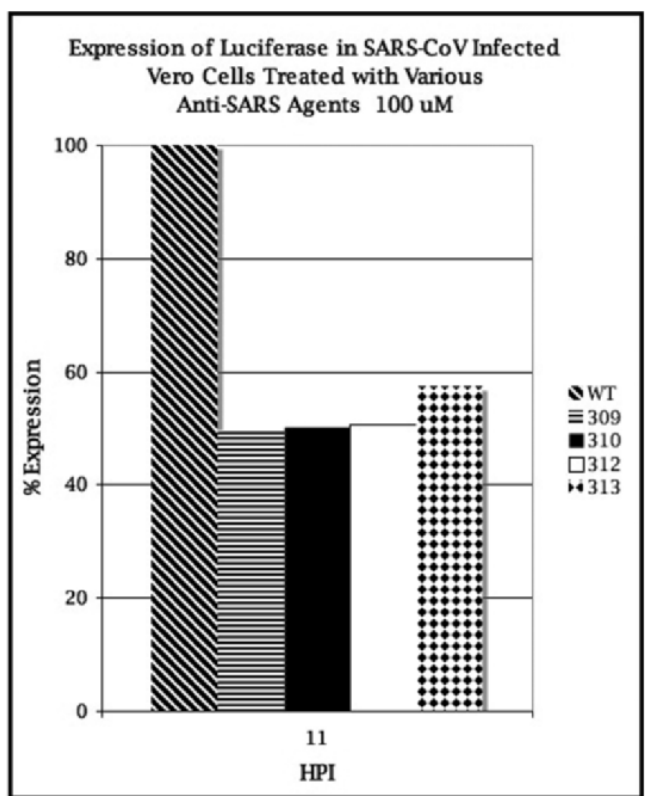

Figure 4. The effect on Luciferase expression in SARS wt-Luc infected VeroE6 cells treated with synthetic compounds designed to inhibit MPro binding.

production. In utilizing this virus, we not only have a sensitive way to evaluate virus growth using Luciferase expression as a proxy, but we also show the importance of the TRS region on subgenomic mRNA production and/or translation.

Our approach offers an alternative to plaque assay analysis in testing the efficiency of anti-SARS agents. The Dual-Glo Luciferase Assay System (Promega) in conjunction with our SARS wt-Luc virus provides a rapid, extremely sensitive, accurate, and highthrough-put screening method.

\section{ACKNOWLEDGMENTS}

This research was funded by NIH grants P01 A1059443 and A1059136.

\section{REFERENCES}

1. T. G. Ksiazek, et al., A novel coronavirus associated with severe acute respiratory syndrome, $N$. Engl J. Med. 348, 1953-1966 (2003).

2. B. Yount, et al., Reverse genetics with a full-length infectious cDNA of severe acute respiratory syndrome coronavirus, Proc. Natl. Acad. Sci. USA 100, 12995-13000 (2003). 